MODELING, IDENTIFICATION AND CONTROL, 1995, vOL. 16, NO. 3, 119-127

doi:10.4173/mic.1995.3.1

\title{
Temperature and flow distributions in planar SOFC stacks
}

\author{
MONICA ØSTENSTAD $\dagger$ and TERJE SIRA $\dagger$ \\ Keywords: SOFC, numerical model, design, simulation
}

Simulation of a planar Solid Oxide Fuel Cell stack requires the solution of the mass balances of the chemical species, the energy balances, the charge balance and the channel flow equations in order to compute the species concentrations, the temperature distributions, the current density and the channel flows. The unit cell geometry can be taken into account by combining detailed modeling of a unit cell with a homogenized model of a whole stack. In this study the effect of the asymmetric temperature distribution on the channel flows in a conventional cross-flow design has been investigated. The bidirectional cross-flow design is introduced, for which we can show more directional temperature and flow distributions.

\section{Introduction}

A fuel cell is an electrochemical reactor which converts the chemical energy of a fuel directly into electric energy. In theory the direct conversion can be made considerably more efficient (i.e. up to 75 per cent) than the 40 per cent maximum now attainable by using the fuel in a heat engine.

During the last three years there have been two large research projects in Norway on the development and demonstration of Solid Oxide Fuel Cells (SOFC). This type of fuel cells has the advantage of being able to use natural gas directly, with a high energy conversion efficiency and a very low content of $\mathrm{NO}_{\mathrm{x}}$ in the exhaust compared to conventional fossil fuelled power plants.

A numerical model for SOFC stacks has been developed as a part of the Norwegian 'NorCell' project. The purpose of the model is to optimize the stack design, to help plan and interpret experiments and to develop good operational strategies. The model computes 3D distributions of the temperature, the current density, the species concentrations and the channel flows (Østenstad 1991).

A 3D model is required in order to compute realistic temperature distributions for stacks in thermal contact with the environment, to compute the effect of the current pickup design and to simulate various thermally integrated reforming designs. Compared with a 2D model, the modifications required due to the vertical dimension are that one set of mass and energy balances for the fuel and air flows has to be computed for each layer, the heat conduction in the vertical direction has to be included, and the electric potential has to be computed at interior points (Selman 1969, 1990).

\section{Homogenization}

A planar SOFC stack can be viewed as if it were composed of identical unit cells as illustrated in Fig. 1. A detailed current computation on this geometry would require that we use a numerical mesh with at least $1000(10 \times 10 \times 10)$ grid points per unit cell.

Received 6 October 1994.

† Institutt for energiteknikk. Kjeller. Norway. 


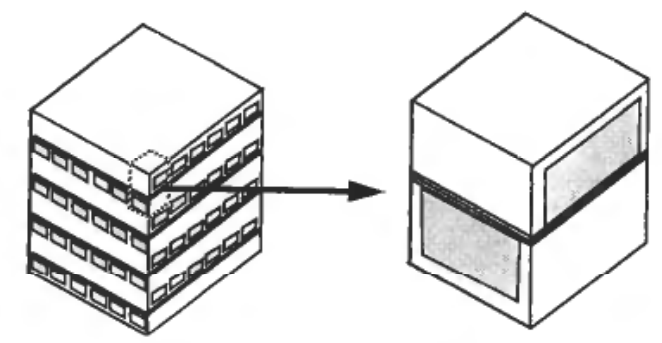

Figure 1. A unit cell of a cross flow stack.

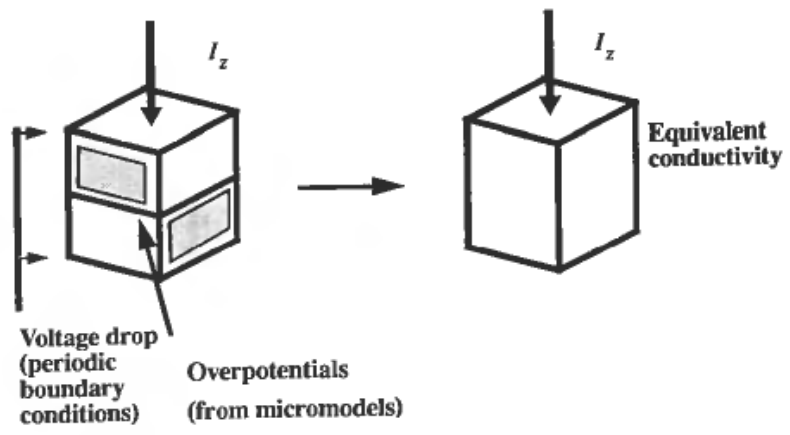

Figure 2. Homogenization of the electric conductivity in the $z$-direction.

Assuming that the outer dimension of the unit cell is $3.3 \times 3.3 \times 3.3 \mathrm{~mm}^{3}$, a $750 \mathrm{~W}$ stack would contain $27000(30 \times 30 \times 30)$ unit cells. Therefore the total size of the numerical problem would be of order $10^{7}$. This problem is however, too large to be solved within reasonable time limits.

Through the use of homogenization techniques the run time can be reduced to an acceptable level (Østenstad 1990). The homogenization transforms the computational problem into one of solving a system of partial differential equations on a homogeneous stack. We can then define a numerical mesh which is independent of the unit cell dimensions, and thus get a computational problem of practical size.

The homogenized model for the SOFC stack is obtained by homogenizing the electrical and thermal conductivities and the Nernst potential (Solheim 1993). Figure 2 illustrates how the homogenized electrical conductivity in the vertical direction can be found empirically, with a detailed model for the unit cell. This model uses a potential difference, $\Delta V$, in the vertical direction along with periodical boundary conditions, the material specific electrical conductivities and the unit cell geometry to compute the total electric current, $I_{z}$ through the cell. The homogenized electrical conductivity, $\sigma_{z}^{h}$, can then be found from the formula:

$$
\sigma_{z}^{h}=\frac{\Delta z}{\Delta x \Delta y} * \frac{I_{z}}{\Delta V}
$$

where $\Delta x, \Delta y$ and $\Delta z$ are the unit cell outer dimensions. The effect of the electrochemical overpotentials may be included in the homogenized conductivities. For a given potential drop the electrical conductivity computed in this manner gives the same total current through the homogeneous cell as a detailed current computation on the true geometry. It also gives the same heat dissipation from ohmic resistance. 
The homogenized electrical conductivities in the other directions and the homogenized thermal conductivities can be found through analogous procedures.

A steady state solution for the electric potential at interior points is found by requiring $\nabla j=0$, where $j$ is the electric current density. In the homogenized model the spatial components of the current density are defined in terms of the homogenized conductivities

$$
\begin{aligned}
& j_{x}=-\sigma_{x}^{h} \frac{\partial \Phi}{\partial x} \\
& j_{y}=-\sigma_{y}^{h} \frac{\partial \Phi}{\partial y} \\
& j_{z}=-\sigma_{z}^{h}\left(\frac{\partial \Phi}{\partial z}-U_{n}^{h} \xi\right)
\end{aligned}
$$

where $\Phi$ is the electric potential, $\xi$ is the number of bipolar plates per unit vertical distance and $U_{n}^{h}$ is the homogenized Nernst potential.

The effect of homogenizing the electrical conductivity and the Nernst potential on the electric potential variation in the vertical direction can be seen by comparing the Figs 3-5. Figure 3 shows the potential variation for a stack of 3 bipolar plates. The potential jumps across the electrolytes are easily identified and the potential drop due to the ohmic resistance are seen to have different slopes in the different materials

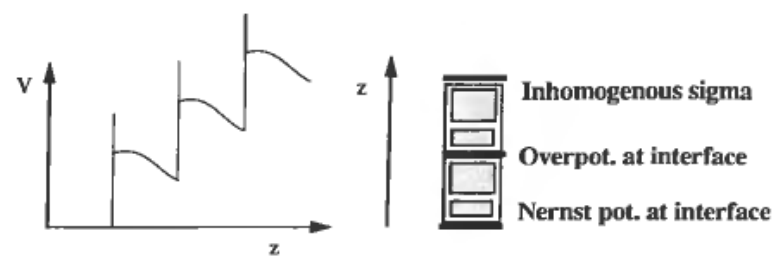

Figure 3. Potential variation in $z$-direction.

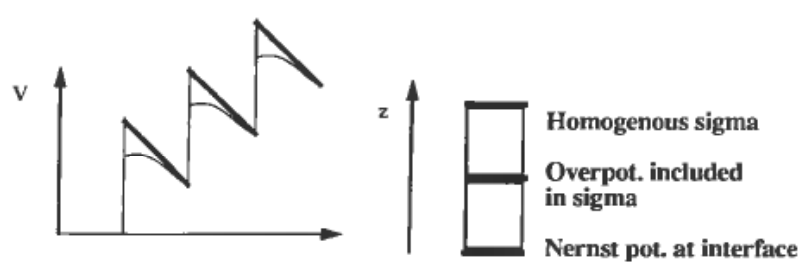

Figure 4. Homogenized electrical conductivities.

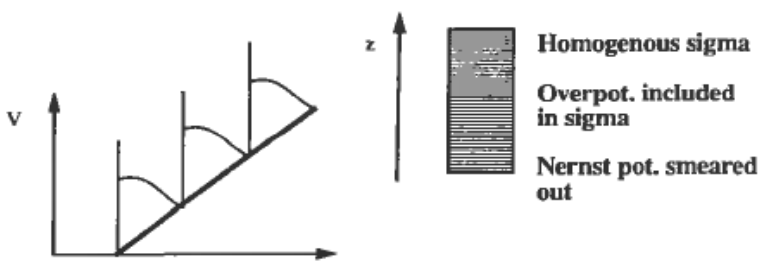

Figure 5. Potential variation with homogenized Nernst potential. 
that the cells are composed of. Figure 4 shows how the potential will vary when homogenized electrical conductivities are used. The potential drop due to ohmic resistance now has a constant slope. Figure 5 shows how the potential will vary in a model where also the Nernst potential has been homogenized. In this case the potential is a linear approximation utilizing the mean of the potential jumps.

Within the framework of the IEA cooperation on modeling and evaluation of Solid Oxide Fuel Cells we have had the opportunity to compare the homogenized 3D model with 2D models that are not based on homogenization (Costa and Arato 1991, Hartvigsen et al. 1992, Rechenauer 1993, Karoliussen 1993). The simulation of one plate (2D) with cross-flow fuelled with hydrogen gives very similar results for the different types of models (Achenbach 1994). This supports the premise that a homogeneous model will give a good approximation to the average behaviour of the cells.

\section{The numerical solution scheme}

The system of coupled differential equations which need to be solved on the homogeneous stack are given in Appendix A. We have discretized the equations by the method of finite volumes, and we have used an upstream approximation on the transport equations and central differences on the elliptic equations.

In solving the system of equations we have iterated on three levels. The outer iteration is between the solution of the channel flow equations, the charge balance equation, and the simultaneous solution of all the other equations. The charge balance equation is solved by the Incomplete Cholesky factorization Conjugated Gradient (ICCG) method.

The reason why the elliptic equation for the solid temperature is solved simultaneously with the transport equations is the strong coupling between the solid temperature and the chemical reaction rates. The coupled system of transport equations could have been solved directly by integrating in the direction of the channel flows. However, since the solid temperature of a given differential volume is coupled to temperatures in all directions, we must iterate on the heat conduction.

Within each differential volume we have used Newton's method for solving the system of non-linear equations (6)-(10) in the Appendix, and in each iteration the linear system for the correction term is solved by Gauss elimination with partial pivoting (Bunch 1987). Experimentally we have seen that the iteration on the non-linearities converges at an acceptable rate. Additionally we have determined that the model is numerically stable in cases where fuel utilizations are close to $100 \%$

The heat conduction iterations consumes the greatest portion of the total computational expense. To improve the convergence rate for this iteration a convergence acceleration routine has been developed which is run between each iteration to find better initial values for the solid temperatures. The convergence acceleration routine has improved the total run-time of the model by a factor of 5 to 10 when simulating a stack fuelled by hydrogen.

\section{Temperature and flow distribution in cross-flow designs}

For planar SOFC stacks the three most common flow configurations are the cross-flow design where the air and the fuel channels are perpendicular, the co-flow design where the channels are parallel and the air and the fuel flow in the same direction, 
and the counter-flow design where the channels are parallel but the air and the fuel flow in opposite directions (Yentekakis 1991).

The main advantage of the cross-flow planar design is the comparative ease of manifolding. One major disadvantage, however, is the fairly uneven temperature and current distribution (Ferguson 1991). The corner closest to the fuel inlet and the air outlet will tend to have a high temperature and a high Nernst potential due to the fresh fuel. This results in a high current density. The opposite corner will have a low current density that is caused by the low Nernst potential in the spent fuel as well as the proximity of the air inlet which will result in a low temperature.

The asymmetry in the temperature distribution will also lead to a spread in the fuel utilization. Since the fuel channels close to the air inlet will, on the average, be colder than the channels close to the air outlet, and since the bulk resistance of the electrolyte decreases markedly with the temperature, we will tend to get a higher utilization in the hotter channels. This effect will be amplified by the fact that the channel flow will decrease with increasing temperature due to the temperature dependence of the viscosity and the density.

One proposed method to remedy this situation is to bidirectionally channel the fuel through the stack. The fresh fuel is only sent through the fuel channels on the air inlet side. The partially spent fuel is then collected in a manifold and returned through the fuel channels on the air outlet side. The main potential advantage with this design is the possibility for a more even current and temperature distribution. The fresh fuel is channelled through the colder part of the stack, while the partly spent fuel is channelled through the hotter part close to the air outlet thus partially balancing the effect of the temperature and the fuel concentration. A more even temperature distribution should also result since the air channels on the average will get a more even heat generation.

In order to study the potential advantages of the bidirectional design, we have compared it with a conventional cross-flow design. The operational parameters and stack properties are the same for the two cases. In order to get the same fuel flow through the stack, the pressure differential over the fuel channels has to be increased.

The cross-flow design had a fairly uneven temperature and current distribution. The maximum temperature was $1279 \mathrm{~K}$, with a $164 \mathrm{~K}$ maximum temperature difference over the stack. The maximum current density was $6189 \mathrm{~A} / \mathrm{m}^{2}$ with a mean value of $2372 \mathrm{~A} / \mathrm{m}^{2}$, and a spread of $5349 \mathrm{~A} / \mathrm{m}^{2}$. The fuel utilization varied from $72 \%$ up to $90 \%$, the mean value being $78.5 \%$. This was partly due to the temperature effect on the fuel flow (about a 5-6\% variation in flow in the channels) and partly due to the temperature dependence of the bulk electrolyte resistance.

The bidirectional case was run with the same input except for the pressure difference over the fuel channels. The most marked difference was a more uniform temperature and current distribution. To illustrate this, Figs 6-9 compare the temperature and the current distributions of the cross-flow and the bidirectional designs. The maximum temperature difference over the stack was reduced to $100 \mathrm{~K}$ while the maximum temperature was reduced from 1279 to $1222 \mathrm{~K}$. The maximum spread in the current density was reduced to $3469 \mathrm{~A} / \mathrm{m}^{2}$, and the maximum current density was reduced from 6189 to $4767 \mathrm{~A} / \mathrm{m}^{2}$. Variation in the fuel utilization was reduced from $15 \%$ to $2 \%$.

The bidirectional design appears to be superior to a conventional cross-flow design from a performance point of view. Even with a $64 \mathrm{~K}$ lower maximum temperature, the bidirectional design had a marginally better performance, with an $80 \%$ mean utilization compared to $78.5 \%$ for the cross-flow design. It is, however, yet to be determined 


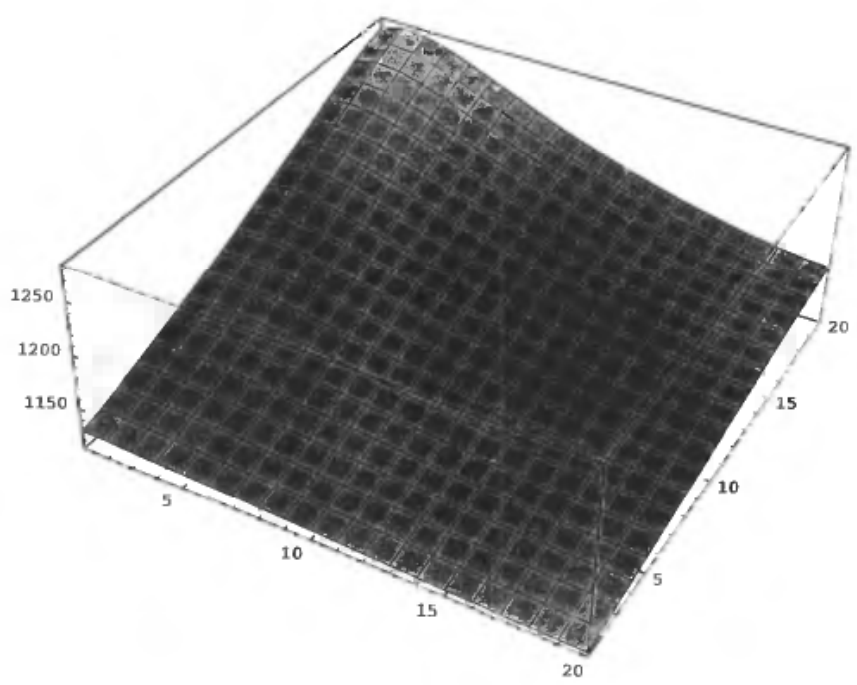

Figure 6. Temperature in crossflow.

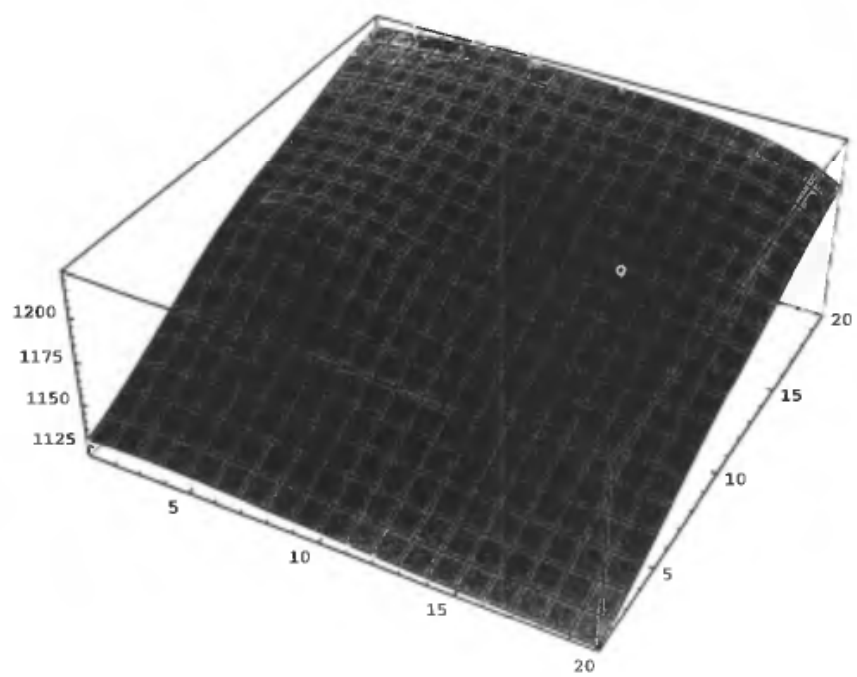

Figure 7. Temperature in biflow.

whether the improved performance justifies the added complexity required for manifolding. Another open issue is how thermal interaction with the environment will affect the advantages of the bidirectional design.

\section{Appendix A: The Model Equations}

The mass balance for oxygen:

$$
\nabla_{2} L=-\frac{\xi}{4 F} j_{z}
$$

The mass balance for fuel component $\gamma$ :

$$
\nabla_{2} K_{\gamma}=\xi A_{\gamma}^{T} \boldsymbol{r}
$$




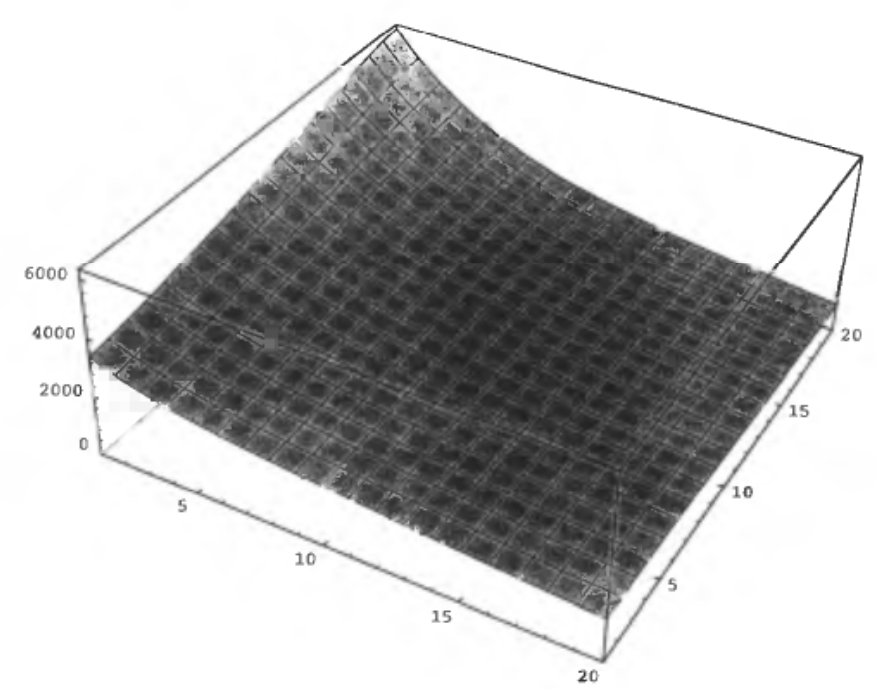

Figure 8. Current density in crossflow.

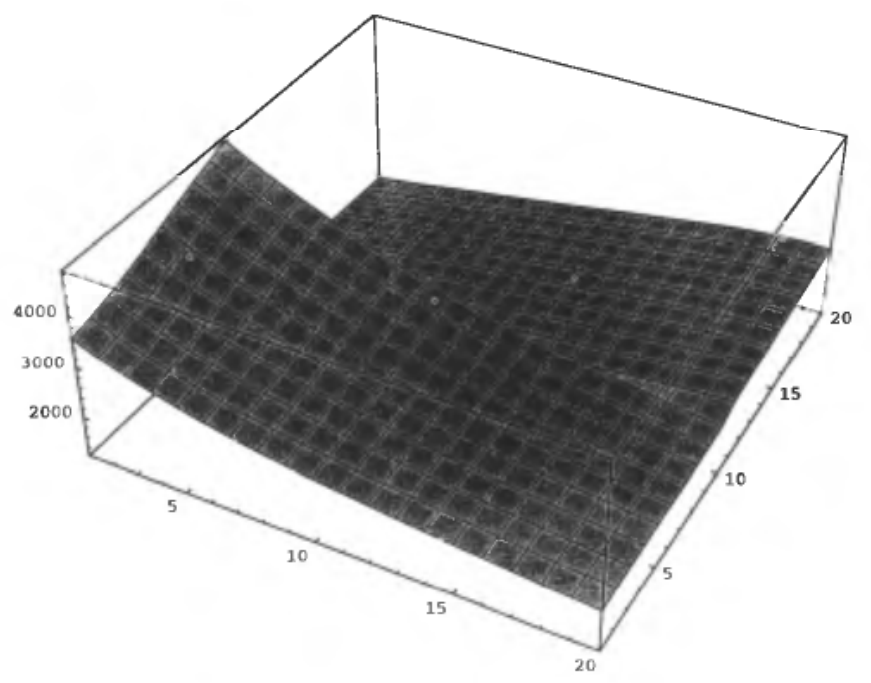

Figure 9. Current density in biflow.

The energy balance in the air flow:

$$
N_{a} C_{p}^{a} \nabla_{2} T_{a}=\alpha_{s}^{a}\left(T_{s}-T_{a}\right)
$$

The energy balance in the fuel flow:

$$
N_{f} C_{p}^{f} \nabla_{2} T_{f}=\alpha_{s}^{f}\left(T_{s}-T_{f}\right)-\xi \sum_{\beta=n_{E C^{+}}}^{n_{E C^{+}+n_{R}}} \Delta H_{\beta} r_{\beta}
$$

The energy balance in the solid:

$$
-\nabla \boldsymbol{q}=\alpha_{s}^{a}\left(T_{a}-T_{s}\right)+\alpha_{s}^{f}\left(T_{f}-T_{s}\right)-\xi \sum_{\beta=1}^{n_{E C}} \Delta H_{\beta} r_{\beta}-j \nabla \Phi
$$


where:

$$
\boldsymbol{q}=\lambda_{x}^{h} \frac{\partial T}{\partial x} \boldsymbol{i}+\lambda_{y}^{h} \frac{\partial T}{\partial y} j+\lambda_{z}^{h} \frac{\partial T}{\partial z} \boldsymbol{k}
$$

The charge balance:

$$
\frac{\partial}{\partial x} \sigma_{x}^{h} \frac{\partial \Phi}{\partial x}+\frac{\partial}{\partial y} \sigma_{y}^{h} \frac{\partial \Phi}{\partial y}+\frac{\partial}{\partial z} \sigma_{z}^{h}\left(\frac{\partial \Phi}{\partial z}-U_{n}^{h} \xi\right)=0
$$

The flow equation for air:

$$
\frac{\partial}{\partial x} g_{x}^{a} \frac{\partial p_{a}}{\partial x}+\frac{\partial}{\partial y} g_{y}^{a} \frac{\partial p_{a}}{\partial y}=S_{a}
$$

The flow equation for fuel:

$$
\frac{\partial}{\partial x} g_{x}^{f} \frac{\partial p_{f}}{\partial x}+\frac{\partial}{\partial y} g_{y}^{f} \frac{\partial p_{f}}{\partial y}=S_{f}
$$

where

$$
\begin{array}{ll}
\nabla & =\frac{\partial}{\partial x} \boldsymbol{i}+\frac{\partial}{\partial y} \boldsymbol{j}+\frac{\partial}{\partial z} \boldsymbol{k} \\
\nabla_{2} & =\frac{\partial}{\partial x} \boldsymbol{i}+\frac{\partial}{\partial y} \boldsymbol{j}
\end{array}
$$

$L \quad$ is the oxygen molar flux density;

$\boldsymbol{K}$ is the vector of fuel component molar flux densities;

$T_{a} \quad$ is the air flow temperature;

$T_{f} \quad$ is the fuel flow temperature;

$T_{s} \quad$ is the temperature of the solid;

$\Phi \quad$ is the electric potential;

$j_{z} \quad$ is the electric current density;

$U_{n}^{h} \quad$ is the homogenized Nernst potential;

$p_{a} \quad$ is the air pressure;

$p_{f} \quad$ is the fuel pressure;

$N_{a} \quad$ is the total molar flux density in the air flow;

$N_{f} \quad$ is the total molar flux density in the fuel flow;

$C_{p}^{a} \quad$ is the heat capacity of air;

$C_{p}^{f} \quad$ is the heat capacity of the fuel;

$A^{T} \quad$ is the transpose of the matrix of stoichiometric coefficients;

$\boldsymbol{r} \quad$ is the vector of chemical reaction rates.

$\Delta H_{\beta}$ is the reaction enthalpy for reaction no. $\beta$;

$\alpha_{s}^{a} \quad$ is the heat transfer coefficient between solid and air;

$\alpha_{s}^{f} \quad$ is the heat transfer coefficient between solid and fuel;

$\lambda^{h} \quad$ is the homogenized thermal conductivity;

$\sigma^{h} \quad$ is the homogenized electrical conductivity;

$g_{a} \quad$ is the air flow conductivity;

$g_{f} \quad$ is the fuel flow conductivity;

$S_{a} \quad$ is the air molar production;

$S_{f} \quad$ is the fuel molar production;

$\xi \quad$ is the number of plates per unit vertical distance;

$n_{E C}$ is the number of electrochemical reactions;

$n_{R} \quad$ is the number of chemical reactions in the fuel flow;

$F \quad$ is the Faraday constant. 


\section{REFERENCES}

ACHENBACH, E. (1994). Status of the IEA-Bench Mark Test 1 on Stack Modelling. The IEA SOFC Newsletter, March 1994, 31-79.

BUNCH, J. R. (1987). The weak and strong stability of algorithms in numerical linear algebra. Linear Algebra and Its Applications, 88, 49-66.

Costa, P., and ARATO, E. (1991). Micro and macro-models for simulation of fuel cell devices. Chimica e l'Industria Milan, Italy. 73, 8-9.

FERGUSON, J. R. (1991). Analysis of temperature and current distributions in planar SOFC designs. Proceedings of the Second International Symposium on Solid Oxide Fuel Cells, Athens, Greece, 2 to 5 July, 1991, pp. 305-312.

HARTVigSen, J., El cells. Proceedings of the Fuel Cell Seminar in Tucson, Arizona, 29 Nov. to 2 Dec. 1992, pp. 532-535.

KAROLIUSSEN, H. (1993). Matematisk modellering av fastoksid brenselcelle. Doktor ingeniøravhandling, Institutt for teknisk elektrokjemi, Trondheim/Norway, November 1993.

Østenstad, M. (1990). Numerical Modelling of SOFC-Design Proposal, IFE/Norway Report no. IFE/KR/F-90/139. November 1990.

ØSTENSTAD, M. (1991). 3-D numerical model for SOFC stacks. Proceedings of 1991 IEA SOFC Workshop, Oslo, Norway, August 18 to 23, 1991, pp. 20-32.

RECHENAUER, C., and ACHENBACH, E. (1993). Three-dimensional mathematical modelling of steady and unsteady state conditions for solid oxide fuel cells. Dissertation, Technische Hochschule Aachen (Germany) April 1993.

Selman, J. R. (1969). How to model NG-fueled SOFC systems. Proceedings of IEA workshop on mathematical modelling, Charmey, Switzerland, July 2 to 6, 1989, pp. 31-63.

Selman, J. R., Herbin, R., FlüCK, M., and Gruber, R. (1990). 2-D \& 3-D Modelling at the Stack Level. Proceedings of IEA Workshop on Modelling and Evaluation of Advanced SOFC, Hertenstein, Switzerland, June 24 to 29, 1990, pp. 17-45.

SOLHEIM, A. (1993). In-plane diffusion in flat-plate solid oxide fuel cells. Proceedings of the third International Symposium on Solid Oxide Fuel Cells, Honolulu, Hawaii, May 1993, pp. 841-850.

YENTEKAKIS, I. V. (1991). Mathematical modelling of cross-flow, counter-flow and cocurrentflow solid oxide fuel cells-Theory and Some Preliminary Experiments. Proceedings of the Second International Symposium on Solid Oxide Fuel Cells, Athens, Greece, 2 to 5 July, 1991, pp. 273-280. 